\title{
Fuzzy Critical Path Method Based on Lexicographic Ordering of Fuzzy Numbers
}

\author{
P. Phani Bushan Rao \\ Department of Engineering Mathematics \\ GIT, GITAM University, Visakhapatnam, India \\ avilok26@yahoo.co.in \\ N. Ravi Shankar \\ Department of Applied Mathematics \\ GIS, GITAM University, Visakhapatnam, India \\ drravi68@gmail.com
}

\begin{abstract}
The Critical Path Method (CPM) is useful for planning and control of complex projects. The CPM identifies the critical activities in the critical path of an activity network. The successful implementation of CPM requires the availability of clear determined time duration for each activity. However, in practical situations this requirement is usually hard to fulfill since many of activities will be executed for the first time. Hence, there is always uncertainty about the time durations of activities in the network planning. This has led to the development of fuzzy CPM. In this paper, we use a Lexicographic ordering method for ranking fuzzy numbers to a critical path method in a fuzzy project network, where the duration time of each activity is represented by a trapezoidal fuzzy number. The proposed method is compared with fuzzy CPM based on different ranking methods of fuzzy numbers. The comparison reveals that the method proposed in this paper is more effective in determining the activity criticalities and finding the critical path. This new method is simple in calculating fuzzy critical path than many methods proposed so far in literature.
\end{abstract}

Keywords: Fuzzy set,Critical path method, Activity network, Trapezoidal fuzzy number, Lexicographical Ordering.

\section{Introduction}

A project network is defined as a set of activities that must be performed according to precedence constraints stating which activities must start after the completion of specified other activities (Durbois et al., 2003). Such a project network can be represented as a directed graph. A path through a project network is one of the routes from the starting node to the ending node. The length of a path is the sum of the durations of the activities on the path. As activities in the network can be carried out in parallel, the minimum time to complete the project is the length of the longest path from the start of the project to its finish. The longest path is therefore the critical path in the network. The project duration equals the length of the longest path through the project network.

In many situations, projects can be complicated and challenging to manage. When the activity times in the project are deterministic and known, CPM has been demonstrated to be a useful tool in managing projects in an efficient manner to meet the challenge (Hilier and Lieberman, 2004). However, there are many cases where the activity times may not be presented in a precise manner. 
To deal quantitatively with imprecise data, the program evaluation and review technique (PERT) (Hilier and Lieberman, 2004) can be employed. However, there are critiques of PERT (Chanas and Zielinski, 2001). An alternative way to deal with imprecise data is to employ the concept of fuzziness (Slyeptosov and Tyshchuk, 2003), whereby the vague activity times can be represented by fuzzy sets. Several studies have investigated the case where activity times in a project are approximately known and are more suitably represented by fuzzy sets rather than crisp numbers (Slyeptosov and Tyshchuk, 2003, Zielinski, 2005, Shankar et al., 2010).

This paper presents another approach, which has not been proposed in the literature so far, to analyze the critical paths in a general project network with fuzzy activity times. We use a Lexicographic ordering ranking method for fuzzy numbers (Farhadina, 2009) to a critical path method for fuzzy project network, where the duration time of each activity in a fuzzy project network is represented by a trapezoidal fuzzy number. We compare this method with various fuzzy critical path methods based on ranking of fuzzy numbers (Shankar et al., 2010, 2010; Yao and Lin, 2000).

\subsection{Literature review of Fuzzy Critical Path Method}

Project scheduling is an important part of project management science. There are several methods for project scheduling such as CPM, PERT and GERT. As there are too many drawbacks involved in these methods, estimating the duration of activities by these methods lack the capability of modelling practical projects. In order to solve these problems, a number of techniques like fuzzy logic, genetic algorithm (GA) and artificial neural network can be considered. A fundamental approach to solve these problems is applying fuzzy sets. Introducing the fuzzy set theory by Zadeh(1965) opened promising new horizons to different scientific areas such as project scheduling. Fuzzy theory (Zimmermann, 1991), with presuming imprecision in decision parameters and utilizing mental models of experts are an approach to adapt scheduling models into reality. To this end, several methods have been developed during the last four decades. The first method called FPERT was proposed by Chanas and Kamburowski (1981). In (Chanas and Kamburowski, 1981) the project completion time is given in the form of a fuzzy set in the time space, thenGazdik (1983) developed a fuzzy network of an a priori unknown project to estimate the activity duration, and used fuzzy algebraic operators to calculate the duration of the project and its critical path. This work is called FNET. An extension of FNET was proposed by Nasution (1994) and Lorterapong and Moselhi (1996). Following on this McCahon (1993), Chang et al. (1995) and, Lin and Yao (2003) presented three methodologies to calculate the fuzzy completion project time. Other researchers such as Kuchta (2001), Yao and Lin (2000), Chanas and Zielinski (2001), Olivers and Robinson (2005) used fuzzy numbers and presented other methods to obtain fuzzy critical paths and critical activities and activity delay. 


\section{Literature review of Ranking Fuzzy Numbers}

As ranking and comparing fuzzy numbers plays a very important role in many fuzzy optimization problems and decision-making procedure, various methods of ranking fuzzy numbers are available in literature and Lexicographical Ordering is one of them. Ranking of fuzzy numbers is not an easy task as fuzzy numbers are represented by possibility distributions and then can overlap with each other. Various approaches on ranking fuzzy numbers have been proposed which can be classified into four major classes; preference relation, fuzzy mean and spread, fuzzy scoring and linguistic expression. Except a few approaches, others are not simple in calculation procedure. There is no single result that can produce a satisfactory result in every situation: some may generate counter-intuitive results and others are not discriminative enough (Bortolan and Degani, 1985). To overcome such problems the method of ranking fuzzy numbers based on lexicographical ordering is introduced by Farhadinia (2009). The method of ranking fuzzy numbers was first proposed by Jain (1976). Since then, a large variety of methods have been developed ranging from the trivial to the complex, including one fuzzy number attribute to many fuzzy number attributes. In a study mentioned by Chen and Hwang (1992), the ranking methods are classified into four major classes which are preference relation, fuzzy mean and spread, fuzzy scoring and linguistic expression. Although the centroid concept has been known and applied in various disciplines since hundreds of years ago, the involvement of centroid concept in ranking fuzzy number only started in 1980 in Yager (1980). Other than Yager (1980), a number of researchers like Murakami et al. (1983), Cheng (1998), Chu and Tsao (2002), Chen and Chen (2003, 2007), Liang and Zhang (2006), Wang and Lee (2008) have also used the centroid concept in developing their ranking index. Each of the researchers presents their own definition of ranking index based on the centroid concept where some of the ranking indices are based on the value of $x$ alone while some are based on the contribution of both $x$ and $y$ values. However, to produce a correct centroid point formula and apply it in the ranking index is also an aim for some researchers like Wang et al. (2006) and Shieh (2007). Their corrected formulae provide a very useful computational support in ranking fuzzy numbers based on the centroid approaches.

The organization of this paper is as follows; in the next section some standard definitions are presented. One of the ranking procedure (Metric distance) of fuzzy numbers is described in section 3 . In the fourth section ranking fuzzy numbers by lexicographic ordering with an example is given. In section 5 , the fuzzy critical path based on lexicographic ordering is described. The final section involves conclusion which tells about the ease of using lexicographic ordering fuzzy numbers in finding fuzzy critical path method.

\section{Preliminaries}

Some preliminary notions, definitions and operations in fuzzy set theory to be used throughout this paper. These are stated as follows: 
Definition 1: Let $X$ be the collection of objects.A fuzzy set $\tilde{A}$ in $X$ is characterized by a membership function $\mu_{\tilde{\mathrm{A}}}: \mathrm{X} \rightarrow[0,1]$ and denoted by $\tilde{\mathrm{A}}=\left\{\left(\mathrm{x}, \mu_{\tilde{\mathrm{A}}}(\mathrm{x})\right) / \mathrm{x} \in \mathrm{X}\right.$.

Definition 2:An $\alpha$-cutor $-\alpha$ levelof the set $\tilde{\mathrm{A}}$, is the $\operatorname{crisp}$ set $[\tilde{\mathrm{A}}]_{\alpha}=\left\{\mathrm{x} \in \mathrm{X} / \mu_{\tilde{\mathrm{A}}}(\mathrm{x}) \geq \alpha\right\}$

Definition 3: The supportof a fuzzy set $\tilde{A}$ is the $\operatorname{crisp} \operatorname{set} \operatorname{supp}(\tilde{A})=$ $\left\{\mathrm{x} \in \mathrm{X} / \mu_{\tilde{\mathrm{A}}}(\mathrm{x})>0\right\}$.

Definition 4: A fuzzy set $\tilde{A}$ is called a fuzzy numberif the following conditions are satisfied:

(i) $\tilde{\mathrm{A}}$ is normal. It means that there exists one $\mathrm{x} \in \mathrm{X}$ such that $\mu_{\tilde{\mathrm{A}}}(x)=1$;

(ii) $\mu_{\tilde{\mathrm{A}}}$ is quasi-convex. It means that for every $\mathrm{x}, \mathrm{y} \in \mathrm{X}$, $\mu_{\tilde{\mathrm{A}}}(\delta \mathrm{x}+(1-\delta) \mathrm{y}) \geq \min \left\{\mu_{\tilde{\mathrm{A}}}(\mathrm{x}), \mu_{\tilde{\mathrm{A}}}(\mathrm{y})\right\}, \delta \in[0,1] ;$

(iii) $\mu_{\tilde{\mathrm{A}}}(x)$ is upper semi-continuous;

(iv) Supp $\tilde{A}$ is bounded in X.

Definition 5:Let $\mathrm{L}, \mathrm{R}:[0, \infty) \rightarrow[0,1]$ be two upper semi-continuous, non-increasing functions satisfying $L(0)=R(0)=1, L(1)=R(1)=0$, invertible on $[0,1]$. Let $a$, $d$ be real positive numbers. The fuzzy number $\tilde{p} \in \mathfrak{R}(R)$ is an LR fuzzy numberif $\tilde{\mathrm{p}} \equiv \mu_{\tilde{\mathrm{p}}}(\mathrm{x})=\left\{\begin{array}{cr}\mathrm{L}\left(\frac{\mathrm{b}-\mathrm{a}}{\mathrm{x}}\right) ; & \mathrm{x} \leq \mathrm{b} \\ 1 ; & \mathrm{b} \leq \mathrm{x} \leq \mathrm{c} \\ \mathrm{R}\left(\frac{\mathrm{x}-\mathrm{c}}{\mathrm{d}}\right) ; & \mathrm{x} \geq \mathrm{c} .\end{array}\right.$

It is symbolically written as $\tilde{p}=(b, c, a, d)_{L R}$ where $b$ and care called the meanvalues satisfying $b \leq c$ and $a, d$ are the left and right spreads, respectively.

Definition 6:An LR fuzzy number $\tilde{p} \in \mathfrak{R}(\mathrm{R})$ is said to be a trapezoidal fuzzy numberif the functions $L$ and $R$ are linear. With this assumption, a real-numbered quadruple $(\mathrm{b}, \mathrm{c}, \mathrm{a}, \mathrm{d})_{\mathrm{LR}}$ represents a trapezoidal fuzzy number. If $\mathrm{b}=\mathrm{c}=\mathrm{p}$ then $(\mathrm{p}, \mathrm{a}, \mathrm{d})_{\mathrm{LR}}$ triple characterizesthe triangular fuzzy number $\tilde{\mathrm{p}} \in \mathfrak{R}(\mathrm{R})$. 


\section{Fuzzy Arithmetic}

Let $\tilde{\mathrm{A}}_{1}$ and $\tilde{\mathrm{A}}_{2}$ be two trapezoidal fuzzy numbers parameterized by the quadruple $\left(a_{1}, b_{1}, c_{1}, d_{1}\right)$ and $\left(a_{2}, b_{2}, c_{2}, d_{2}\right)$ respectively. Then, Fuzzy numbers addition $\oplus:\left(\mathrm{a}_{1}, \mathrm{~b}_{1}, \mathrm{c}_{1}, \mathrm{~d}_{1}\right) \oplus\left(\mathrm{a}_{2}, \mathrm{~b}_{2}, \mathrm{c}_{2}, \mathrm{~d}_{2}\right)=\left(\mathrm{a}_{1}+\mathrm{a}_{2}, \mathrm{~b}_{1}+\mathrm{b}_{2}, \mathrm{c}_{1}+\mathrm{c}_{2}, \mathrm{~d}_{1}+\mathrm{d}_{2}\right)$ Fuzzy numbers subtraction $\Theta$ :

$$
\left(a_{1}, b_{1}, c_{1}, d_{1}\right) \Theta\left(a_{2}, b_{2}, c_{2}, d_{2}\right)=\left(a_{1}-d_{2}, b_{1}-c_{2}, c_{1}-b_{2}, d_{1}-a_{2}\right) \text {. }
$$

\section{Fuzzy critical path method based on metric distance ranking}

\subsection{Metric distance ranking}

Chen and Cheng [34] proposed a metric distance method to rank fuzzy numbers. Let $\tilde{\mathrm{A}}$ and $\tilde{\mathrm{B}}$ be two fuzzy numbers defined as follows:

$$
\begin{aligned}
& f_{\tilde{\mathrm{A}}}(x)=\left\{\begin{array}{l}
f_{\tilde{\mathrm{A}}}^{L}(x), x<m_{\tilde{\mathrm{A}}} \\
f_{\tilde{\mathrm{A}}}^{R}(x), x \geq m_{\tilde{\mathrm{A}}}
\end{array}\right. \\
& f_{\tilde{\mathrm{B}}}(x)=\left\{\begin{array}{l}
f_{\tilde{\mathrm{B}}}^{L}(x), x<m_{\tilde{\mathrm{B}}} \\
f_{\tilde{\mathrm{B}}}^{R}(x), x \geq m_{\tilde{\mathrm{B}}}
\end{array}\right.
\end{aligned}
$$

where $m_{\tilde{\mathrm{A}}}$ and $m_{\tilde{\mathrm{B}}}$ are the mean of $\tilde{\mathrm{A}}$ and $\tilde{\mathrm{B}}$. The metric distance between $\tilde{\mathrm{A}}$ and $\tilde{B}$ can be calculated as follows:

$$
D(\tilde{\mathrm{A}}, \tilde{\mathrm{B}})=\left[\int_{0}^{1}\left(g_{\tilde{\mathrm{A}}}^{L}(y)-g_{\tilde{\mathrm{B}}}^{L}(y)\right)^{2} d y+\int_{0}^{1}\left(g_{\tilde{\mathrm{A}}}^{R}(y)-g_{\tilde{\mathrm{B}}}^{R}(y)\right)^{2} d y\right]^{\frac{1}{2}},
$$

where $g_{\tilde{\mathrm{A}}}^{L}, g_{\tilde{\mathrm{A}}}^{R}, g_{\tilde{\mathrm{B}}}^{L}$ and $g_{\tilde{\mathrm{B}}}^{R}$ are the inverse functions of $f_{\tilde{\mathrm{A}}}^{L}, f_{\tilde{\mathrm{A}}}^{R}, f_{\tilde{\mathrm{B}}}^{L}$ and $f_{\tilde{\mathrm{B}}}^{R}$, respectively.

If the fuzzy number $\tilde{B}=0$, then the metric distance between $\tilde{A}$ and 0 is calculated as follows:

$$
D(\tilde{\mathrm{A}}, 0)=\left[\int_{0}^{1}\left(g_{\tilde{\mathrm{A}}}^{L}(y)\right)^{2} d y+\int_{0}^{1}\left(g_{\tilde{\mathrm{A}}}^{R}(y)\right)^{2} d y\right]^{\frac{1}{2}}
$$

The larger the value of $\mathrm{D}(\tilde{\mathrm{A}}, 0)$, the better the ranking of $\tilde{\mathrm{A}}$.

According to Chen and Cheng (2005), a trapezoidal fuzzy number $\tilde{\mathrm{A}}=$ $\left(a_{1}, a_{2}, a_{3}, a_{4}\right)$ can be approximated as a symmetry fuzzy number $\mathbf{S}[\mu, \sigma]$, where 
$\mu$ denotes the mean of $\tilde{\mathrm{A}}$ and $\sigma$ denotes the standard deviation of $\tilde{\mathrm{A}}$, and the membership function of $\tilde{A}$ is defined as follows:

$$
f_{\tilde{\mathrm{A}}}(x)=\left\{\begin{array}{l}
\frac{x-(\mu-\sigma)}{\sigma}, \text { if } \mu-\sigma \leq x \leq \mu \\
\frac{(\mu+\sigma)-x}{\sigma}, \text { if } \mu \leq x \leq \mu+\sigma
\end{array}\right.
$$

where $\mu$ and $\sigma$ are calculated as follows:

$$
\begin{aligned}
\sigma & =\frac{2\left(a_{4}-a_{1}\right)+a_{3}-a_{2}}{4}, \\
\mu & =\frac{a_{1}+a_{2}+a_{3}+a_{4}}{4},
\end{aligned}
$$

If $a_{2}=a_{3}$, then $\tilde{\mathrm{A}}$ becomes a triangular fuzzy number, where $\tilde{\mathrm{A}}=\left(a_{1}, a_{2}, a_{4}\right)$ and $\mu$ and $\sigma$ can be calculated as follows:

$$
\begin{aligned}
\sigma & =\frac{a_{4}-a_{1}}{2}, \\
\mu & =\frac{a_{1}+2 a_{2}+a_{4}}{4}
\end{aligned}
$$

The inverse functions $g_{\tilde{\mathrm{A}}}^{L}$ and $g_{\tilde{\mathrm{A}}}^{R}$ of $f_{\tilde{\mathrm{A}}}^{L}$ and $f_{\tilde{\mathrm{A}}}^{R}$ respectively, are shown as follows:

$$
\begin{aligned}
& g_{\tilde{\mathrm{A}}}^{L}(y)=(\mu-\sigma)+\sigma \times y \\
& g_{\tilde{\mathrm{A}}}^{R}(y)=(\mu+\sigma)-\sigma \times y
\end{aligned}
$$

\subsection{Fuzzy CPM Based on Metric Distance [8]}

The operation time for each activity in the fuzzy project network is characterized as a positive trapezoidal fuzzy number. In accordance with CPM, the forward pass yields the fuzzy earliest-start and earliest-finish times:

$$
\begin{aligned}
& \tilde{E}_{i}^{s}=\max _{j \in P(i)}\left\{\tilde{E}_{j}^{s} \oplus \tilde{t}_{j}\right\} \\
& \tilde{E}_{i}^{f}=\tilde{E}_{i}^{s} \oplus \tilde{t}_{i}
\end{aligned}
$$

where $\quad \tilde{E}_{i}^{s}$ is the fuzzy earliest -start time with $\tilde{E}_{A}^{s}=(0,0,0)$ at the initial node $i=\mathrm{A}, \tilde{E}_{i}^{f}$ is the fuzzy earliest finish time with $\tilde{E}_{Z}^{f}$ equal to the fuzzy project network completion time $\tilde{T}$ at the ending node $i=\mathrm{Z}, \mathrm{P}(i)$ is the set of predecessors for activity $i$, and $\tilde{t}_{i}$ is the operation time for activity $i$. The 
backward pass is performed to calculate the fuzzy latest-start and latest-finish times:

$$
\begin{aligned}
& \tilde{L}_{i}^{f}=\min _{j \in P(i)}\left\{\tilde{L}_{j}^{f} \Theta \tilde{t}_{j}\right\} \\
& \tilde{L}_{i}^{s}=\tilde{L}_{i}^{f} \Theta \tilde{t}_{i}
\end{aligned}
$$

where $\tilde{L}_{i}^{f}$ is the fuzzy latest-finish time with $\tilde{L}_{z}^{f}=\tilde{T}$ at the end node $i=Z, \tilde{L}_{i}^{s}$ is the fuzzy latest start time and $\mathrm{S}(i)$ is the set of successors for activity $i$. Once $\tilde{E}_{i}^{s}$, $\tilde{E}_{i}^{f}, \tilde{L}_{i}^{s}$ and $\tilde{L}_{i}^{f}$ have been determined for the $i^{\text {th }}$ activity, the fuzzy float time is either

$$
\begin{aligned}
T_{i}^{F}=\tilde{L}_{i}^{s} \Theta \tilde{E}_{i}^{s} \\
\quad \text { or } \\
T_{i}^{F}=\tilde{L}_{i}^{f} \Theta \tilde{E}_{i}^{f}
\end{aligned}
$$

We can easily compute the fuzzy float times of all activities in a project network using the method by Shankar et al. (2010).

\section{Example:}

Fig.1 shows the network representation of a fuzzy project network. Table I represents the total float of each activity in a fuzzy project network.

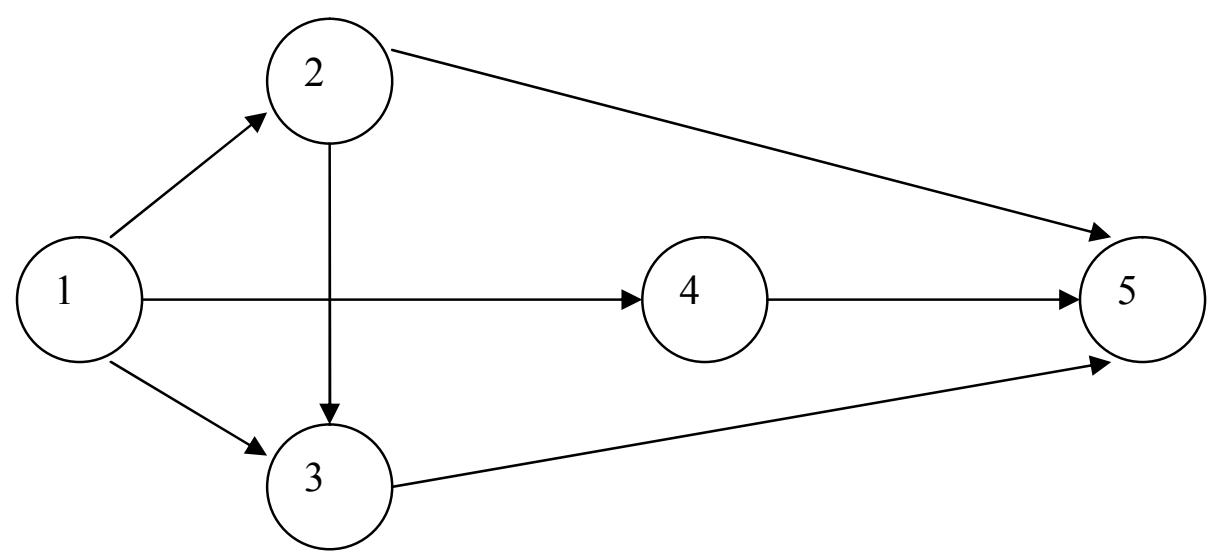

Fig. 1 A fuzzy project network 
Table I: Total float of each activity in the fuzzy project network

\begin{tabular}{|l|l|l|l|}
\hline Activity & $\begin{array}{c}\text { Fuzzy Activity } \\
\text { time }\end{array}$ & $\begin{array}{c}\text { Total float } \\
(\mathbf{a}, \mathbf{b}, \mathbf{c}, \mathbf{d})\end{array}$ & $\begin{array}{c}\text { Total float } \\
(\mathbf{b}, \mathbf{c}, \mathbf{b}-\mathbf{a}, \mathbf{c}-\mathbf{d})\end{array}$ \\
\hline $1-2$ & $(10,15,15,20)$ & $(-160,-60,60,160)$ & $(-60,60,100,-100)$ \\
\hline $1-3$ & $(30,40,40,50)$ & $(-130,-35,75,170)$ & $(-35,75,120,200)$ \\
\hline $2-3$ & $(30,40,40,50)$ & $(-160,-60,60,160)$ & $(-60,60,100,-100)$ \\
\hline $1-4$ & $(15,20,25,30)$ & $(-110,-20,95,185)$ & $(-20,95,90,-90)$ \\
\hline $2-5$ & $(60,100,150,180)$ & $(-100,-10,100,190)$ & $(-10,100,90,-90)$ \\
\hline $3-5$ & $(60,100,150,180)$ & $(-160,-60,60,160)$ & $(-60,60,100,-100)$ \\
\hline $4-5$ & $(60,100,150,180)$ & $(-110,-20,95,185)$ & $(-20,95,90,-90)$ \\
\hline
\end{tabular}

The possible paths of fuzzy project network (Fig.1) are 1-2-3-5, 1-2-5, 1-3-5 and 1-4-5.

Metric distance rank of total fuzzy slack time for each path in fuzzy project network (Fig. 1) are computed and presented in table II.

\section{Case (i) Path: 1-2-3-5}

Total fuzzy slack time for the path $1-2-3-5$ is $\quad(a, b, c, d)=(-480,-180,180,480)$.

Then $(b, c, b-a, c-d)=(-180,180,300,-300)$

Let $\tilde{A}=\left(a_{1}, a_{2}, a_{3}, a_{4}\right)=(-180,180,300,-300)$

$\sigma=\frac{2\left(a_{4}-a_{1}\right)+a_{3}-a_{2}}{4}=-30$

$\mu=\frac{a_{1}+a_{2}+a_{3}+a_{4}}{4}=0$

$g_{\tilde{\mathrm{A}}}^{L}(y)=(\mu-\sigma)+\sigma \times y=30(-\mathrm{y}+1)$

$g_{\tilde{\mathrm{A}}}^{R}(y)=(\mu+\sigma)-\sigma \times y=30(-1+\mathrm{y})$

$D(\tilde{\mathrm{A}}, 0)=\left[\int_{0}^{1}\left(g_{A}^{L}(y)\right)^{2} d y+\int_{0}^{1}\left(g_{A}^{R}(y)\right)^{2} d y\right]^{\frac{1}{2}}=24.49$

Case (ii) Path: 1-2-5

Total fuzzy slack time for the path $1-2-5$ is $\quad(a, b, c, d)=(-260,-70,160,350)$

Then $(b, c, b-a, c-d)=(-70,160,190,-190)$

Let $\tilde{\mathrm{A}}=\left(\mathrm{a}_{1}, \mathrm{a}_{2}, \mathrm{a}_{3}, \mathrm{a}_{4}\right)=(-70,160,190,-190)$

$\sigma=\frac{2\left(a_{4}-a_{1}\right)+a_{3}-a_{2}}{4}=-52.5$

$\mu=\frac{a_{1}+a_{2}+a_{3}+a_{4}}{4}=22.5$

$g_{\tilde{\mathrm{A}}}^{L}(y)=(\mu-\sigma)+\sigma \times y \quad=75-52.5 \mathrm{y}$

$g_{\tilde{\mathrm{A}}}^{R}(y)=(\mu+\sigma)-\sigma \times y=-30+52.5 \mathrm{y}$ 


$$
D(\tilde{\mathrm{A}}, 0)=\left[\int_{0}^{1}\left(g_{A}^{L}(y)\right)^{2} d y+\int_{0}^{1}\left(g_{A}^{R}(y)\right)^{2} d y\right]^{\frac{1}{2}}=53.38
$$

\section{Case (iii) Path: 1-3-5}

Total fuzzy slack time for the path $1-3-5$ is $\quad(a, b, c, d)=(-290,-95,135,330)$

Then $(b, c, b-a, c-d)=(-95,135,195,-195)$

Let $\tilde{\mathrm{A}}=\left(\mathrm{a}_{1}, \mathrm{a}_{2}, \mathrm{a}_{3}, \mathrm{a}_{4}\right)=(-95,135,195,-195)$

$\sigma=\frac{2\left(a_{4}-a_{1}\right)+a_{3}-a_{2}}{4}=-35$

$\mu=\frac{a_{1}+a_{2}+a_{3}+a_{4}}{4}=10$

$g_{\tilde{\mathrm{A}}}^{L}(y)=(\mu-\sigma)+\sigma \times y=45-35 \mathrm{y}$

$g_{\tilde{\mathrm{A}}}^{R}(y)=(\mu+\sigma)-\sigma \times y=-25+35 y$

$D(\tilde{\mathrm{A}}, 0)=\left[\int_{0}^{1}\left(g_{A}^{L}(y)\right)^{2} d y+\int_{0}^{1}\left(g_{A}^{R}(y)\right)^{2} d y\right]^{\frac{1}{2}}=31.58$

\section{Case (iv) Path: 1-4-5}

Total fuzzy slack time for the path $1-4-5$ is $(a, b, c, d)=(-220,-40,190,370)$

Then $(b, c, b-a, c-d)=(-40,190,180,-180)$

Let $\tilde{\mathrm{A}}=\left(\mathrm{a}_{1}, \mathrm{a}_{2}, \mathrm{a}_{3}, \mathrm{a}_{4}\right)=(-40,190,180,-180)$

$\sigma=\frac{2\left(a_{4}-a_{1}\right)+a_{3}-a_{2}}{4}=-72.5$

$\mu=\frac{a_{1}+a_{2}+a_{3}+a_{4}}{4} \quad=37.5$

$g_{\tilde{\mathrm{A}}}^{L}(y)=(\mu-\sigma)+\sigma \times y=110-72.5 \mathrm{y}$

$g_{\tilde{\mathrm{A}}}^{R}(y)=(\mu+\sigma)-\sigma \times y=-35+72.5 \mathrm{y}$

$D(\tilde{\mathrm{A}}, 0)=\left[\int_{0}^{1}\left(g_{A}^{L}(y)\right)^{2} d y+\int_{0}^{1}\left(g_{A}^{R}(y)\right)^{2} d y\right]^{\frac{1}{2}}=79.48$.

Table II: Metric distance rank for each path in fuzzy project network

\begin{tabular}{|l|l|l|}
\hline Path & $\begin{array}{c}\text { Total fuzzy slack time } \\
(\mathbf{a}, \mathbf{b}, \mathbf{c}, \mathbf{d})\end{array}$ & $\begin{array}{c}\text { Metric } \\
\text { distance rank }\end{array}$ \\
\hline $1-2-3-5$ & $(-480,-180,180,480)$ & 24.49 \\
\hline $1-2-5$ & $(-260,-70,160,350)$ & 53.38 \\
\hline $1-3-5$ & $(-290,-95,135,330)$ & 31.58 \\
\hline $1-4-5$ & $(-220,-40,190,370)$ & 79.48 \\
\hline
\end{tabular}


Here, the path having minimum rank is $1-2-3-5$. Therefore, the required critical path for the fuzzy project is 1-2-3-5.

\section{Fuzzy Lexicographical Ordering}

Although problem of ordering of fuzzy numbers has been discussed broadly so far and many approaches have been extensively proposed, they contained some shortcomings and in some situations they may fail to exhibit the consistency of human intuition. Also most of the existing approaches are not simple in calculation procedure. In order to overcome the mentioned problems, especially the complexity of the computational procedures, it is here presented a ranking method based on lexicographical ordering. A question to ask is why lexicographic order is implemented to compare fuzzy numbers. This is due to it providing decision makers with a simple and efficient algorithm that formulates an ordering founded on precedence and also the lexicographic order is a total order on ground terms when the precedence is total. In other words, any two fuzzy numbers $\tilde{p}, \tilde{\mathrm{q}} \in \mathfrak{R}(R)$ are comparable in the sense that one has either $\tilde{p}<\tilde{\mathrm{q}}$ or $\tilde{p}>\tilde{\mathrm{q}}$ or $\tilde{p} \approx \tilde{\mathrm{q}}$. Before proceeding to present the main results, a number of definitions are required at this stage.

Definition 7:Let $\tilde{p} \in \mathfrak{R}(R)$ be a fuzzy number. Define

(i) $G(\tilde{\mathrm{p}})=\inf \{x \in \operatorname{supp}(\tilde{\mathrm{p}}) ; \tilde{\mathrm{p}}(x)=1\}$,

(ii) $H(\tilde{\mathrm{p}})=\inf \{\operatorname{supp}(\tilde{\mathrm{p}})\}$,

(iii) $\mathrm{I}(\tilde{\mathrm{p}})=|\operatorname{supp}(\tilde{\mathrm{p}})|$,

(iv) $\mathrm{J}(\tilde{\mathrm{p}})=\int \tilde{\mathrm{p}} d x$

$(\mathrm{v}) \mathrm{K}(\tilde{\mathrm{p}})=(\mathrm{G}(\tilde{\mathrm{p}}), \mathrm{H}(\tilde{\mathrm{p}}), \mathrm{I}(\tilde{\mathrm{p}}), \mathrm{J}(\tilde{\mathrm{p}}))$.

Definition 8:For $X, Y \in R^{n}$, the lexicographical ordering on $R^{n}$ is defined $\prec_{\text {lex }}$ means that $X \prec_{l e x} Y$ if and only if $x_{i}<y_{i}$ for $1 \leq i \leq n$ and $x_{j}=y_{j}$ for $j<i$.

Furthermore means that $X \prec_{l e x} Y$ or $X=Y$.

On the basis of the latter definitions, the following fuzzy lexicographic order is established on $\Re(R)$ :

(i) $\tilde{\mathrm{p}}<\tilde{\mathrm{q}}$ if and only if $\mathrm{K}(\tilde{\mathrm{p}}) \prec_{\text {lex }} \mathrm{K}(\tilde{\mathrm{q}})$,

(ii) $\tilde{\mathrm{p}} \leq \tilde{\mathrm{q}}$ if and only if $\mathrm{K}(\tilde{\mathrm{p}}) \leqslant_{\operatorname{lex}} \mathrm{K}(\tilde{\mathrm{q}})$,

(iii) $\tilde{\mathrm{p}} \approx \tilde{\mathrm{q}}$ if and only if $\mathrm{K}(\tilde{\mathrm{p}}) \preccurlyeq_{l e x} \mathrm{~K}(\tilde{\mathrm{q}})$ and $\mathrm{K}(\tilde{\mathrm{p}}) \succcurlyeq_{\text {lex }} \mathrm{K}(\tilde{\mathrm{q}})$ if and only if $\mathrm{K}(\tilde{\mathrm{p}})=\mathrm{K}(\tilde{\mathrm{q}})$ 
It is to be noted that unlike different types of the existing ranking orders, the fuzzy lexicographic order is so easy to handle the calculations. As particular case, if $\mathrm{p}$ in parametricrepresentation is given by $(b, c, a, d)_{L R}$ then

$$
\mathrm{G}(\tilde{\mathrm{p}})=\mathrm{b}, \mathrm{H}(\tilde{\mathrm{p}})=\mathrm{b}-\mathrm{a}, \mathrm{I}(\tilde{\mathrm{p}})=\mathrm{c}-\mathrm{b}+\mathrm{a}+\mathrm{d} \quad \text { and } \quad \mathrm{J}(\tilde{\mathrm{p}})=\mathrm{c}-\mathrm{b}+\frac{1}{2}(\mathrm{a}+\mathrm{d})
$$

Another advantage which arises from this ordering is a simple and fast algorithm to determine stepwise the precedence ordering of the fuzzy numbers. However, the algorithm may terminate successfully at step one while the comparison is complete.

\section{Working Rule (Fuzzy lexicographic ordering)}

Step 1: consider two trapezoidal fuzzy numbers $\tilde{\mathrm{p}}$ and $\tilde{\mathrm{q}}$ (LR type) and find $\mathrm{G}, \mathrm{H}$, $\mathrm{I}$ and $\mathrm{J}$ for $\tilde{\mathrm{p}}$ and $\tilde{\mathrm{q}}$ using definition 8 ..

Step 2: Compare $G(\tilde{p})$ and $G(\tilde{q})$. If $G(\tilde{p})=G(\tilde{q})$ then go to Step 3. Otherwise stop and the larger $\mathrm{G}($ num) is the larger corresponding to the fuzzy number num .

Step 3: Compare $\mathrm{H}(\tilde{\mathrm{p}})$ and $\mathrm{H}(\tilde{\mathrm{q}})$. If $\mathrm{H}(\tilde{\mathrm{p}})=\mathrm{H}(\tilde{\mathrm{q}})$ then go to Step 4. Otherwise stop and the larger $\mathrm{H}$ (num) is the larger corresponding to the fuzzy number num .

Step 4: Compare $(\tilde{p})$ and $I(\tilde{q})$. If $I(\tilde{p})=I(\tilde{q})$ then go to Step 5. Otherwise stop and the larger I( num) is the larger corresponding to the fuzzy number num .

Step 5: Compare $J(\tilde{p})$ and $J(\tilde{q})$. If $J(\tilde{p})=J(\tilde{q})$ then stop and in this case $\tilde{p} \approx \tilde{q}$. Otherwise the larger $\mathrm{J}($ num) is the larger corresponding fuzzy number num .

\section{Numerical Examples:}

In this section, the aim is to demonstrate that the results of the fuzzy lexicographic order are generally more reasonable than the outcomes when ranked with the other approaches.

\section{Example 1:}

Consider the two fuzzy numbers $\tilde{\mathrm{p}}=(2,2,0.1,0.1)_{\mathrm{LR}}$ and $\tilde{\mathrm{q}}=(3,3,0.9,1)_{\mathrm{LR}}$ $G(p)=2$ and $G(\tilde{q})=3$, step 1 of working rule 4 satisfies

One can observe that $\tilde{\mathrm{p}}$ should be intuitively smaller than $\tilde{q}$. The ranking outcome with the CV index proposed Cheng (2004) is $\tilde{\mathrm{p}} \succ \tilde{\mathrm{q}}$ which is not real. It 
is easy to see that the fuzzy lexicographic order leads to $\tilde{p} \prec \tilde{q}$ at the first step of working rule resulting $G(\tilde{p})=2<G(\tilde{q})=3$.

Therefore, $\tilde{\mathrm{p}}<\tilde{\mathrm{q}}$

\section{Example 2:}

Let $\tilde{\mathrm{p}}=(6,6,1,1)_{\mathrm{LR}}, \tilde{\mathrm{q}}=(6,6,0.1,1)_{\mathrm{LR}}$ and $\tilde{\mathrm{r}}=(6,6,0,1)_{\mathrm{LR}}$

$\mathrm{G}(\mathrm{p})=6, \mathrm{G}(\mathrm{q})=6, \mathrm{G}(\mathrm{r})=6$, step 1 of working rule fails.

$\mathrm{H}(\tilde{\mathrm{p}})=5, \mathrm{H}(\tilde{\mathrm{q}})=5.9, \mathrm{H}(\mathrm{r})=6$, step 2 of working rule satisfies.

Therefore, $\tilde{\mathrm{p}}<\tilde{\mathrm{q}}<\tilde{\mathrm{r}}$.

\section{Example 3:}

Let $\tilde{\mathrm{p}}=(0.4,0.7,0.1,0.2)_{\mathrm{LR}}, \tilde{\mathrm{q}}=(0.7,0.7,0.4,0.2)_{\mathrm{LR}}$ and $\tilde{\mathrm{r}}=(0.7,0.7,0.2,0.2)_{\mathrm{LR}}$

$\mathrm{G}(\mathrm{p})=0.4, \mathrm{G}(\mathrm{q})=0.7, \mathrm{G}(\mathrm{r})=3$, step 1 of working rule fails.

$\mathrm{H}(\mathrm{p})=0.3, \mathrm{H}(\mathrm{q})=0.3, \mathrm{H}(\mathrm{r})=0.5$, step 2 of working rule fails.

$\mathrm{I}(\tilde{\mathrm{p}})=0.6, \mathrm{I}(\tilde{\mathrm{q}})=0.6, \tilde{\mathrm{I}}(\tilde{\mathrm{r}})=0.4$, step 3 or working rule fails.

$\mathrm{J}(\tilde{\mathrm{p}})=0.45, \mathrm{~J}(\tilde{\mathrm{q}})=0.3, \tilde{\mathrm{J}}(\tilde{\mathrm{r}})=0.2$, step 4 of working rule satisfies.

Therefore $\tilde{p}>\tilde{q}>\tilde{r}$.

\section{Example 4:}

Let $\tilde{p}=(0.5,0.5,0.2,0.2)_{\mathrm{LR}}, \tilde{\mathrm{q}}=(0.5,0.8,0.2,0.1)_{\mathrm{LR}}$ and $\tilde{\mathrm{r}}=(0.5,0.5,0.2,0.4)_{\mathrm{LR}}$

$\mathrm{G}(\tilde{\mathrm{p}})=0.5, \mathrm{G}(\tilde{\mathrm{q}})=0.5, \mathrm{G}(\tilde{\mathrm{r}})=0.5$, step 1 of working rule fails.

$\mathrm{H}(\tilde{\mathrm{p}})=0.3, \mathrm{H}(\tilde{\mathrm{q}})=0.3, \mathrm{H}(\tilde{\mathrm{r}})=0.3$, step 2 of working rule fails.

$\mathrm{I}(\tilde{\mathrm{p}})=0.4, \mathrm{I}(\tilde{\mathrm{q}})=0.6, \mathrm{I}(\tilde{\mathrm{r}})=0.6$, step 3 or working rule fails.

$\mathrm{J}(\tilde{\mathrm{p}})=0.2, \mathrm{~J}(\tilde{\mathrm{q}})=0.45, \tilde{\mathrm{J}(\tilde{\mathrm{r}})}=0.3$, step 4 of working rule satisfies.

Therefore $\tilde{\mathrm{p}}<\tilde{\mathrm{r}}<\tilde{\mathrm{q}}$. 


\section{Fuzzy CPM Based on Lexicographic ordering of Fuzzy Numbers}

The operation time for each activity in the fuzzy project network is characterized as a positive trapezoidal fuzzy number. In accordance with CPM, the forward pass yields the fuzzy earliest-start and earliest-finish times:

$$
\tilde{\mathrm{E}}_{\mathrm{i}}^{\mathrm{s}}=\succ_{\mathrm{lex}}\left\{\tilde{\mathrm{E}}_{\mathrm{i}}^{\mathrm{s}} \oplus \tilde{\mathrm{P}(\mathrm{i})} \tilde{\mathrm{t}}_{\mathrm{j}}\right\} \text { and } \tilde{\mathrm{E}}_{\mathrm{i}}^{\mathrm{f}}=\tilde{\mathrm{E}}_{\mathrm{i}}^{\mathrm{s}} \oplus \tilde{\mathrm{t}_{\mathrm{i}}}
$$

where $\tilde{\mathrm{E}}_{\mathrm{i}}^{\mathrm{s}}$ is the fuzzy earliest start time with $\tilde{\mathrm{E}}_{\mathrm{A}}{ }^{\mathrm{s}}=(0,0,0)$ at the initial node $\mathrm{i}=\mathrm{A}$, $\tilde{\mathrm{E}}_{\mathrm{i}}{ }^{\mathrm{f}}$ is the fuzzy earliest finish time with $\tilde{\mathrm{E}}_{z}{ }^{\mathrm{f}}$ equal to the fuzzy project network completion time $\tilde{T}$ at the ending node $i=z, P(i)$ is the set of predecessors for activity $i$, and $\tilde{t}_{i}$ is the operation time for activity $i$.

The backward pass is performed to calculate the fuzzy latest-start and latestfinish times.

$$
\tilde{\mathrm{L}}_{\mathrm{i}}^{\mathrm{f}}=\prec_{\substack{\text { lex } \\ \mathrm{j} \in S(\mathrm{i})}}\left\{\tilde{\mathrm{L}}_{\mathrm{j}}^{\mathrm{f}} \oplus \tilde{\mathrm{t}_{\mathrm{j}}}\right\} \text { and } \tilde{\mathrm{L}}_{\mathrm{i}}^{\mathrm{s}}=\tilde{\mathrm{L}}_{\mathrm{i}} \Theta \tilde{\mathrm{t}_{\mathrm{i}}}
$$

Where $\tilde{\mathrm{L}}_{\mathrm{i}}^{\mathrm{f}}$ is the fuzzy latest-finish time with $\tilde{\mathrm{L}}_{z}^{\mathrm{f}}=\tilde{\mathrm{T}}$ at the end node $i=z, \tilde{\mathrm{L}}_{\mathrm{i}}^{\mathrm{s}}$ is the fuzzy latest -start time and $S(i)$ is the set of successors for activity $i$.

Once $\tilde{\mathrm{E}}_{i}^{\mathrm{s}}, \tilde{\mathrm{E}}_{\mathrm{i}}{ }^{\mathrm{f}}, \tilde{\mathrm{L}}_{\mathrm{i}}^{\mathrm{s}}, \tilde{\mathrm{L}}_{\mathrm{i}}^{\mathrm{f}}$ have been determined for the $\mathrm{i}^{\text {th }}$ activity, the fuzzy float time is either

$$
T_{i}^{F}=\tilde{L}_{i}^{s} \Theta \tilde{E}_{i}^{s} \text { Or } T_{i}^{F}=\tilde{L}_{i}^{f} \Theta \tilde{E}_{i}{ }^{f}
$$

We can easily compute the fuzzy float times of all activities in a project network.

\section{Example:}

The possible paths of fuzzy project network (Fig.1) are 1-2-3-5, 1-2-5, 1-3-5 and 1-4-5.

Lexicographical Ordering of total fuzzy slack time for each path in fuzzy projectnetwork (Fig.1) are computed and presented in table III.

Table III: Fuzzy slack time for each path with LR-Representation

\begin{tabular}{|l|l|l|}
\hline Path & $\begin{array}{c}\text { Total fuzzy slack time } \\
(\mathbf{a}, \mathbf{b}, \mathbf{c}, \mathbf{d})\end{array}$ & LR-Representation \\
\hline $1-2-3-5$ & $(-480,-180,180,480)$ & $\tilde{\mathrm{p}}=(-180,180,300,300)$ \\
\hline $1-2-5$ & $(-260,-70,160,350)$ & $\tilde{\mathrm{q}}=(-70,160,190,190)$ \\
\hline $1-3-5$ & $(-290,-95,135,330)$ & $\tilde{\mathrm{r}}=(-95,135,195,195)$ \\
\hline
\end{tabular}




\begin{tabular}{|l|l|l|}
\hline $1-4-5$ & $(-220,-40,190,370)$ & $\tilde{\mathrm{s}}=(-40,190,180,180)$ \\
\hline
\end{tabular}

From the above table the Lexicographical Ordering of total fuzzy slack time for each path in fuzzy project network is $\tilde{\mathrm{p}} \prec \tilde{\mathrm{r}} \prec \tilde{\mathrm{q}} \prec \tilde{\mathrm{s}}$. Here, the path having minimum Lexicographical ordering is $1-2-3-5$. Therefore, the required critical path for the fuzzy project is 1-2-3-5.

\section{Conclusion}

A new analytical method using lexicographical ordering ranking for finding critical path in a fuzzy project network has been proposed. We have computed lexicographic ordering rank of total fuzzy slack time for each path in fuzzy project network to find the critical path in a fuzzy project network. A numerical example has particularly provided to explain the proposed procedure in detail and this method is compared with fuzzy critical path method based on signed distance ranking of fuzzy numbers. The comparison reveals that the method proposed in this paper is more effective in determining the activity criticalities, finding the critical path. This method also shows that the ease of calculations when compared with metric distance ranking. We find that the Lexicographic ordering is simple in evaluation and gives a quick comparison of fuzzy numbers rather than the metric distance ranking of fuzzy numbers.It has been verified that this method is simple in calculating fuzzy critical path method than many methods proposed so far in literature.

\section{References}

1. A.I.Slyeptosov, T.A. Tyshchuk,(2003). Fuzzy temporal characteristics of operationsfor project management on the network models basis, European Journal ofOperations Research 147: 253 -265.

2. B. Farhadina,(2009). Ranking Fuzzy Numbers Based on Lexicographical Ordering, International Journal of Applied Mathematics and Computer Sciences, 5:248-251.

3. Bortolan, G., Degani, R. (1985). A review of some methods for ranking fuzzy subsets, Fuzzy Sets and Systems, 15:1-19.

4. Chanas, S. and Zielinski, P. (2001). Critical Path Analysis in the network with Fuzzy Activity Times, Fuzzy Sets and Systems, 122:195-204.

5. Chang, S., T.Sujimura,Y. and Tazawa, T.(1995). An efficient approach for large scale project planning based on Fuzzy Delphi Method, Fuzzy Sets and Systems, 76:277-288.

6. Chen LS, Cheng $\mathrm{CH}$ (2005). Selecting IS personnel using ranking fuzzy number by metric distance method. European Journal of Operational research 160 (3): 803-820.

7. Chen, S.J. and L. Hwang,C. (1992). Fuzzy multiple attribute decision making methods and applications. In Lecture Notes in Economics and Mathematical Systems, New York: Springer. 
8. Chen, S.J., and Chen, S.M. (2003). A new method for handling multicriteria fuzzydecision making problems using FN-IOWA operators, Cybernatics and Systems,34:109-137.

9. Chen, S.J., and Chen, S.M. (2007). Fuzzy risk analysis based on the ranking of generalized trapezoidal fuzzy numbers, Applied Intelligence, 26:1-11.

10. Cheng, C.H. (1998). A new approach for ranking fuzzy numbers by distance method,Fuzzy Sets and Systems, 95:307-317.

11. Chu, T.C., and Tsao, C.T. (2002). Ranking fuzzy numbers with an area between the centroid point and original point, Computers and Mathematics with Applications, 43:111-117.

12. D.Dubois, H. Fargier, V.Galvagonon, (2003). On latest starting times and floats in task networks with ill-known durations, European Journal of Operational Research 147: 266-310.

13. F.S.Hillier, G.J.Lieberman, (2001). Introduction to Operations Research, seventh edition, McGraw-Hill.

14. I. Gazdik,(1983). Fuzzy Network Planning-FNET, IEEE transactions on reliability, R-32(3):304-313.

15. Jain, R. (1976). Decision making in the presence of fuzzy variables, IEEE transactions on Systems, Man and Cybernetics, 6:698-703.

16. Kuchta, D. (2001). Use of Fuzzy numbers in project risk (criticality) assessment, International Journal of Project Management, 19:305-310.

17. Liang, C., Wu, J. and Zhang, J. (2006, June 21-23). Ranking indices and rules for fuzzy numbers based on gravity center point. Paper presented at the $6^{\text {th }}$ World Congress on Intelligent Control and Automation, Dalian, China.

18. Lin, F.T., Yao, J.S. (2003). Fuzzy Critical Path Method based on signeddistance ranking and statistical confidence- interval estimates, Journal of Super Computing, 24(3):305-325.

19. Lorterapong, P. And Moselhi, O.,(1996). Project-network scheduling using Fuzzy Set theory, Journal of construction engineering and management, ASCE, 122(4):308-318.

20. McCahon, C.S.(1993). Using PERT as an approximation of fuzzy projectnetworkanalysis IEEE transactions on Engineering Management, 40(2):146-153.

21. Murakami, S., Maeda, S. and Imamura, S. (1983). Fuzzy decision analysis on the development of centralized regional energy control; system. Paper presented at the IFAC symposium on Fuzzy Inform. Knowledge Representation and decision Analysis.

22. Nasution S.H.(1994). Fuzzy Critical Path Method, IEEE transactions on Systems, Man and Cybernetics, 24:48-47.

23. NazirahRamli and DaudMohamad (2009). A Comparative Analysis of Centroid Methods in Ranking Fuzzy Numbers, European Journal of Scientific Research, 28(3): 492-501. 
24. Oliveros, A., Robinson, A. (2005). Fuzzy logic approach for activity delay analysis andschedule up dating, Journal of Construction Engineering and Management, 131(1):42-51.

25. P.Zielinski,(2005). On computing the latest starting times and floats of activities in anetwork with imprecise durations, Fuzzy Sets and Systems 150: 53-76.

26. Ravi Shankar, N., Sireesha, V., PhaniBushanRao, P., (2010). An AnalyticalMethod for Finding Critical Path in a Fuzzy Project Network, International Journal of Contemporary Mathematical Sciences, 5 (20): 953-962.

27. Ravi Shankar,N., Sireesha,V., SrinivasaRao, K., Vani,N., (2010). Fuzzy Critical Path Method based on Metric Distance Ranking of Fuzzy Numbers, Int. Journal of Math. Analysis, 4(20):995-1006.

28. S.Chanas, P.Zielinski, (2001). Critical path analysis in the network with fuzzy activity times, Fuzzy sets and Systems 122: 195-204.

29. Shieh, B.S. (2007). An approach to centroids of fuzzy numbers, International Journal of Fuzzy Systems, 9(1):51-54.

30. Stefan Chanas and Jerzy Kamburowski,(1981). The use of fuzzy variables in PERT, Fuzzy sets and Systems, 5(1):11-19.

31. Wang, Y.H., Yang, J.B., Xu, D.L. and Chin, K.S. (2006). On the centroids of fuzzynumbers, fuzzy Sets and Systems, 157:919-926.

32. Wang, Y.J., and Lee, H.S. (2008). The revised method of ranking fuzzy numberswith an area between the centroid and original points, Computers and Mathematics with applications, 55:2033-2042.

33. Yager, R.R. (1980). On a general class of fuzzy connectives, Fuzzy Sets and Systems, 4(6):235-242.

34. Yao, J.S., Lin, F.T. (2000). Fuzzy Critical Path Method based on signed distanceRanking of fuzzy numbers, IEEE transactions on Systems, Man and Cybernetics,30(1):76-82.

35. Zadeh, L.A.(1965). Fuzzy sets, Information and control 8: 338-353.

36. Zimmermann HJ (1991). Fuzzy set theory and it's applications. Kluwer publications, The Netherlands. 